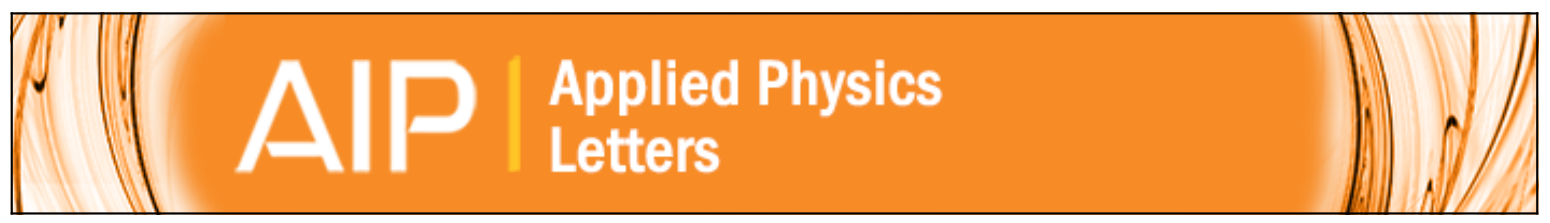

\title{
Electronic property of Na-doped epitaxial graphenes on SiC
}

Seon-Myeong Choi and Seung-Hoon Jhi

Citation: Applied Physics Letters 94, 153108 (2009); doi: 10.1063/1.3120274

View online: http://dx.doi.org/10.1063/1.3120274

View Table of Contents: http://scitation.aip.org/content/aip/journal/apl/94/15?ver=pdfcov

Published by the AIP Publishing

\section{Articles you may be interested in}

A density functional theory study of epitaxial graphene on the $(3 \times 3)$-reconstructed C-face of SiC Appl. Phys. Lett. 102, 093101 (2013); 10.1063/1.4794176

Observation of the quantum Hall effect in epitaxial graphene on $\mathrm{SiC}(0001)$ with oxygen adsorption Appl. Phys. Lett. 100, 253109 (2012); 10.1063/1.4729824

Changes in structural and electronic properties of graphene grown on $6 \mathrm{H}-\mathrm{SiC}(0001)$ induced by $\mathrm{Na}$ deposition

J. Appl. Phys. 111, 083711 (2012); 10.1063/1.4704396

Substrate doping effects on Raman spectrum of epitaxial graphene on SiC

J. Appl. Phys. 107, 034305 (2010); 10.1063/1.3283922

Erratum: "Electronic property of Na-doped epitaxial graphenes on SiC" [Appl. Phys. Lett.94, 153108 (2009)] Appl. Phys. Lett. 95, 089901 (2009); 10.1063/1.3213392

Want to publish your paper in the \#1 MOST CITED journal in applied physics?

With Applied Physics Letters, you can.

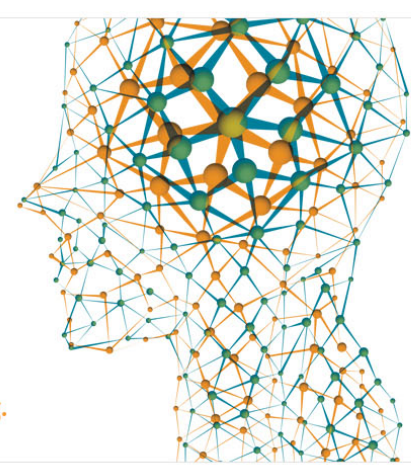




\title{
Electronic property of Na-doped epitaxial graphenes on SiC
}

\author{
Seon-Myeong Choi and Seung-Hoon $\mathrm{Jhi}^{\mathrm{a})}$ \\ Department of Physics, Pohang University of Science and Technology, Pohang 790-784, Republic of Korea
}

(Received 12 January 2009; accepted 26 March 2009; published online 16 April 2009)

The electronic property of epitaxial graphenes with $\mathrm{Na}$ adsorption or intercalation is studied with the use of pseudopotential density functional method. It is found that the charge transfer and the $\mathrm{Na}$ binding energy show strong coverage dependence. Calculated energetics shows that Na prefers the intercalation between the buffer and top graphene layers to the adsorption on top graphene layer. The buffer layer is inert to $\mathrm{Na}$ adsorption on top graphene layer but it is charged when $\mathrm{Na}$ atoms are intercalated. This indicates that the conduction of epitaxial graphenes can be affected significantly by $\mathrm{Na}$ intercalation. (C) 2009 American Institute of Physics. [DOI: 10.1063/1.3120274]

Many interesting physics of graphene have been reported and attracted great attention of scientists and engineers in various fields. Graphene is truly a two-dimensional (2D) structural material with linear electronic bands near the Fermi level. ${ }^{1,2}$ The charge carriers thus behave like massless Dirac fermions with definite chirality in 2D. This unique structural and electronic property has stimulated many interesting experiments such as quantum Hall effect. ${ }^{3,4}$ Constrict nanostructures of graphene known as graphene nanoribbons also exhibit very rich and interesting physics such as sizeand orientation-dependent electronic structures. ${ }^{5}$ In parallel, there have been great efforts to develop electronic devices that utilize the large carrier mobility and inertness to impurity scattering. For practical application of graphenes to electronic devices, it is necessary to resolve several key issues such as reproducible growth of single graphene layers, gap opening, and doping of the graphenes. ${ }^{6}$ Single layer graphenes are now obtained by mechanical peeling of graphite $^{7}$ or by epitaxial growth on $6 \mathrm{H}-\mathrm{SiC}$ surfaces. ${ }^{8}$ Atomic adsorption is a straightforward way to change the carrier density or modify the electronic structure of graphene. Alkaline metals are well-known elements that adsorb on ${ }^{9,10}$ or intercalate into graphite ${ }^{11}$ to donate electrons. In this paper we studied the electronic property of epitaxial graphene with $\mathrm{Na}$ adsorption or intercalation using pseudopotential density functional method.

Computation was carried out using the pseudopotential density functional method with plane-wave basis set. ${ }^{12}$ The exchange-correlation of electrons was treated within the generalized gradient approximation as implemented by Perdew-Berke-Enzelhof. ${ }^{13}$ The cutoff energy for the expansion of wave functions and potentials in the plane-wave basis was chosen to be $400 \mathrm{eV}$, and the Brillouin zone sampling was done with the Monkhorst-Pack special $k$-point method with a grid of $5 \times 5 \times 1$ for the $\sqrt{3} \times \sqrt{3} R 30^{\circ}$ phase. We used the projector augmented wave pseudopotentials as provided by the Vienna Ab-initio Simulation Package. ${ }^{14}$ The atomic relaxation was carried out until the Helmann-Feynman forces were less than $0.02 \mathrm{eV} / \AA$. The vacuum layer in the supercell used in our calculations is set to be $8 \AA$, which is enough to minimize the artificial interlayer interaction.

First, we studied the sodium atom adsorption on freestanding single layer graphenes. Figures 1(a) and 1(b) sum-

${ }^{a)}$ Electronic mail: jhish@postech.ac.kr. marizes our calculations of charge transfer from sodium to graphene and the $\mathrm{Na}$ adsorption energy at varying $\mathrm{Na}$ coverage. Among the top of carbon, the bridge site, and the hexagon center, sodium atoms bind to the hexagon center most strongly (calculated adsorption energies are 0.52, 0.43, and $0.67 \mathrm{eV}$, respectively). The $1 \mathrm{ML}$ of $\mathrm{Na}$ on graphene is defined as one $\mathrm{Na}$ atom per five $\mathrm{C}$ atoms. ${ }^{10}$ Our calculations [Fig. 1(a)] show that the amount of charge transfer per $\mathrm{Na}$ atom is almost one electron until 0.1 ML and then decreases as an inverse of $\rho_{\mathrm{Na}}$ ( $\mathrm{Na}$ density or coverage) at higher cov-

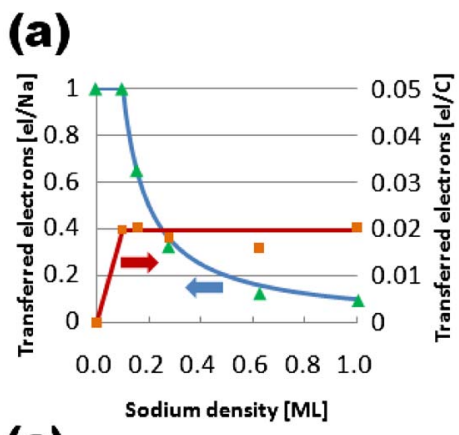

(b)

(c)

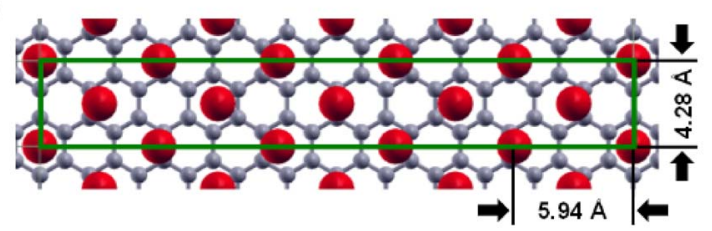

(d)

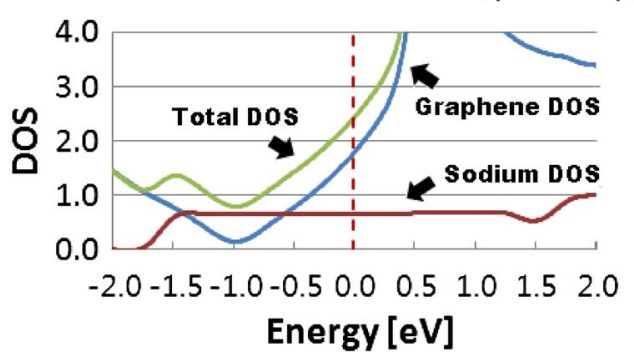

FIG. 1. (Color online) (a) Calculated charge transfer from Na to graphene per sodium atom (green triangles) or per carbon atom (orange squares) with analytic fitting curves (see the text) and (b) the adsorption energy of $\mathrm{Na}$ at varying sodium coverage on graphene. (c) Atomic structure of sodium (denoted by large red balls) on graphene (small gray balls) at $1.04 \mathrm{ML}$ coverage. Calculated $\mathrm{Na}$ interatomic distance is also shown. (d) Corresponding density of states (DOS) for the structure shown in (c). Partial DOS obtained by projecting onto graphene (blue) and $\mathrm{Na}$ (red) are also shown for comparison. 
erage. This $1 / \rho_{\mathrm{Na}}$ behavior is obtained by maximizing the energy gain from the transfer, $E_{\mathrm{ad}}=\left(E_{s}-\bar{E}\right) n+c n^{2} \rho_{\mathrm{Na}}$, where $E_{s}, n$, and $\bar{E}$ are the energy level of $\mathrm{Na} 3 s$ state [or - $(\mathrm{Na}$ ionization energy)], the electron transfer per $\mathrm{Na}$, and the average electronic energy of transferred electrons in graphene (this depends on $n$ and $\rho_{\mathrm{Na}}$ ), respectively. The last term in $E_{\mathrm{ad}}$ is the Coulomb energy with $c$ being a constant. Assuming a linear density of states (DOS) of graphene $\left[\sim D\left(E-E_{0}\right)\right.$ with $D$ and $E_{0}$ being constants] to express $\bar{E}$ as a function of $n$ and $\rho_{\mathrm{Na}}$, the energy gain can be written as

$$
E_{\mathrm{ad}}=\left(E_{s}-E_{0}\right) n-\frac{2}{3} \sqrt{\frac{2 \rho_{\mathrm{Na}}}{D}} n^{3 / 2}+c n^{2} \rho_{\mathrm{Na}} .
$$

The maximum condition of $E_{\text {ad }}$ with respect to $n$ gives the $1 / \rho_{\mathrm{Na}}$ behavior (or the constant charge transfer per carbon). As the coverage increases further, the inter-Na interaction first lowers $\mathrm{Na} 3 s$ level by the effect of ionic Na potential and next broadens the level to form a metallic band by wave-function overlap. This lowering and then rising of the energy level of $\mathrm{Na} 3 s$ state deviates the charge transfer per $\mathrm{Na}$ from $1 / \rho_{\mathrm{Na}}$, making a dip at around $0.6 \mathrm{ML}$ in the charge transfer per carbon. The dependency of calculated adsorption energies of $\mathrm{Na}$ on coverage also fits into the above equations at low coverage $(<0.28 \mathrm{ML})$ as it decreases almost as $1 / \rho_{\mathrm{Na}}$. At higher coverage, $\mathrm{Na}$ atoms start to form metallic bonding with each other and the overall (adsorption) energy appears to increase resultantly. Figure 1(c) is the atomic arrangement for the 1.04 ML sodium on top of graphene and Fig. 1(d) shows the partial and total DOS for this configuration. From calculated DOS, we observe that the free electron states (flat Na partial DOS) are not empty, which indicates that $\mathrm{Na}$ metallic bonds are formed at large sodium coverage.

Next, we studied $\mathrm{Na}$ adsorption on graphenes epitaxially grown on $\mathrm{SiC}$ (0001) surface. It is well-known that $\mathrm{SiC}$ (0001) surface upon heating turns into carbon-rich surface, namely, $6 \sqrt{3} \times 6 \sqrt{3} \mathrm{R} 30^{\circ}$ buffer layer (or reconstructed substrate) and then single layer graphenes at further heating. ${ }^{15}$ Instead of using the large size $6 \sqrt{3} \times 6 \sqrt{3} \mathrm{R} 30^{\circ}$ structure, here we carried out calculations of $\mathrm{Na}$ adsorption on a smallersized structure of $\sqrt{3} \times \sqrt{3} \mathrm{R} 30^{\circ}$ shown in Fig. 2 for computational convenience (we expect that the adsorption properties of $\mathrm{Na}$ is not sensitive to the size of the structure because of free electronlike nature of $\mathrm{Na}$ atom). As not only single graphene layers but also buffer layers are exposed to $\mathrm{Na}$ deposition in experiment, ${ }^{16}$ we studied $\mathrm{Na}$ adsorption for all available configurations: on the buffer layer (without upper graphene layer), on top-most graphene layer and the intercalation between graphene and buffer layers.

As Na coverage of $1 \mathrm{ML}$ is not commensurate with underlying $\mathrm{SiC}$, we chose $0.63 \mathrm{ML}$ coverage with $\mathrm{Na}$ atoms adsorbing on the hexagon centers. Figure 2 shows the atomic arrangement of the clean or Na-adsorbed (0.63 ML) buffer layer (without upper graphene layer) together with calculated band structures for corresponding configurations. The bufferlayer $\pi$ bands are completely empty without $\mathrm{Na}$ adatoms [Fig. 2(b)] in agreement with previous calculations. ${ }^{17} \mathrm{We}$ also observe a flatband near the Fermi level that comes from the top $\mathrm{Si}$ dangling bonds. ${ }^{18}$ For $\mathrm{Na}$ adsorption, two inequivalent hexagon centers are available due to underlying $\mathrm{SiC}$ substrates. Calculated adsorption energies on the two hexagon centers [Figs 2(c) and 2(d)] are 0.84 and $0.78 \mathrm{eV}$,
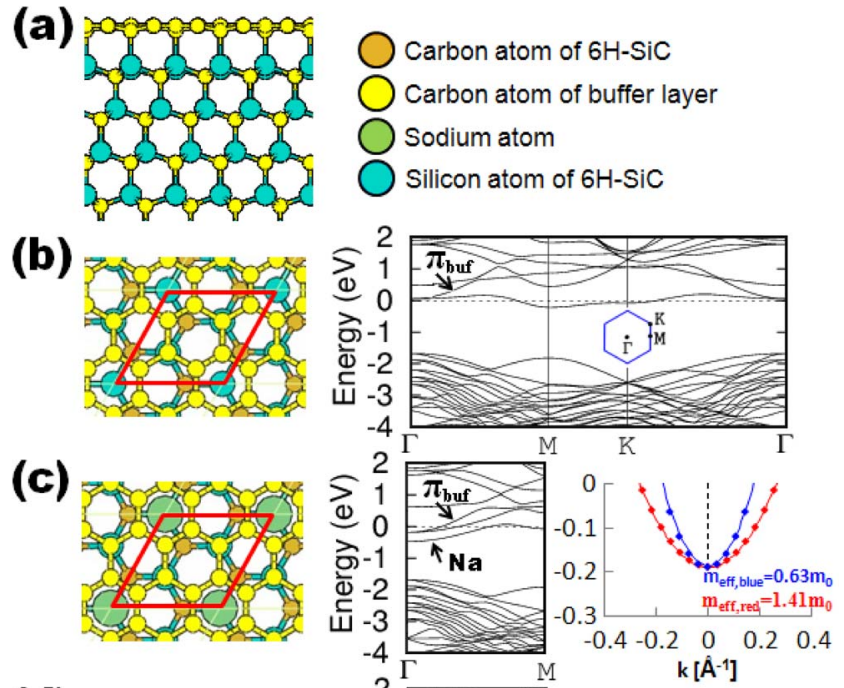

(d)
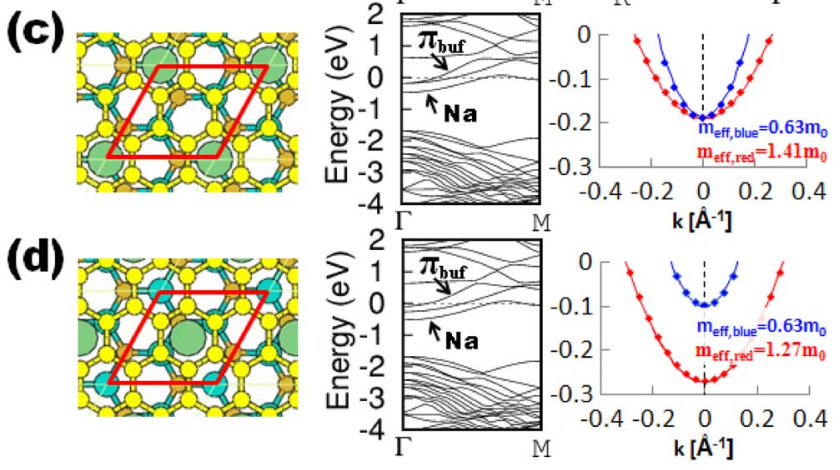

FIG. 2. (Color online) (a) The side view of the $\sqrt{3} \times \sqrt{3} \mathrm{R} 30^{\circ}$ buffer layer on top of $\mathrm{SiC}$ (0001) surface. The small light and dark yellow (color online) circles denote carbon atoms in the buffer and $\mathrm{SiC}$ substrate, respectively. $\mathrm{Na}$ and $\mathrm{Si}$ atoms are drawn as large green and medium blue circles, respectively. (b) The top view and calculated band structure of the buffer layer. We observe that the buffer layer $\pi$ bands are not occupied. [(c) and (d)] show the $\mathrm{Na}$ adsorption on the buffer layer with $0.63 \mathrm{ML}$ coverage for two inequivalent hexagon centers due to underlying substrates. The configuration shown in (c) is energetically more favorable by about $0.06 \mathrm{eV}$ than that in (d). The full band structure near the Fermi level is shown on the right together with the enlarged view of buffer layer $\pi$ bands near $\Gamma$-point. The symmetry of the buffer layer is broken in (d) due to the underlying substrates, which leads to the splitting of the buffer layer $\pi$ bands.

respectively. Two parabolic $\pi$ bands from the buffer layer near the $\Gamma$-point are now partially occupied by the electrons transferred from sodium atoms. The underlying $\mathrm{SiC}$ substrates also affect the degeneracy of the buffer layer $\pi$ bands. Figure 2(c) shows that the degeneracy at $\Gamma$-point is preserved because the original atomic symmetry is still preserved even upon $\mathrm{Na}$ adsorption. Whereas, the bands are split for the $\mathrm{Na}$ adsorption shown in Fig. 2(d). While the Na adsorption shown in Fig. 2(c) is energetically more favorable than in Fig. 2(d), the energy difference is so small that we expect a broadening in the $\pi$ band from the buffer layer.

Next, we studied the adsorption and the intercalation of $\mathrm{Na}$ in graphene-buffer layer structure on $\mathrm{SiC}$ substrate. Figure 3 shows the graphene- $\sqrt{3} \times \sqrt{3} \mathrm{R} 30^{\circ}$ buffer layer with or without $\mathrm{Na}$ adsorption or intercalation between buffer and top graphene layers. Without $\mathrm{Na}$ [Fig. 3(a)], the graphene $\pi$ band shifts down due to charge transfer from buffer to top graphene layer, which is consistent with previous reports. ${ }^{18,19}$ When $\mathrm{Na}$ adsorbs on top graphene layer [Fig. 3(b)], the graphene $\pi$ band shifts down further due to additional charge transfer from $\mathrm{Na}$ atoms. We also observe a parabolic band near $\Gamma$-point that originates from $\mathrm{Na} 3 s$ states. This behavior is very similar to that for the $\mathrm{Na}$ adsorption on isolated graphene. However, the adsorption energy in Fig. 3(b) case is calculated to be about $0.73 \mathrm{eV}$, which is larger than that in isolated graphene of about $0.61 \mathrm{eV}$, for $0.63 \mathrm{ML} N \mathrm{Na}$ cover- 
(a)

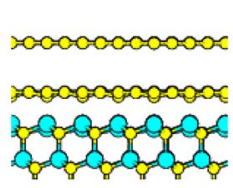

(b)

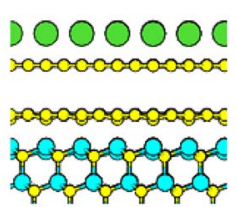

(c)

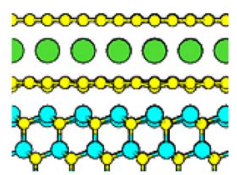

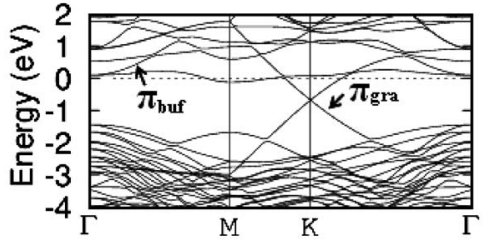
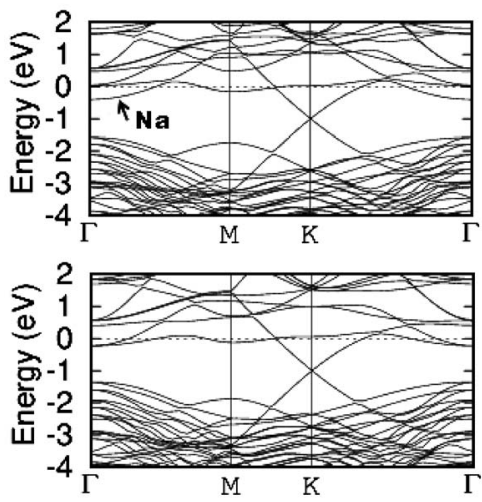

FIG. 3. (Color online) Side view of atomic structures of epitaxial graphenes on $\mathrm{SiC}$ substrate and corresponding band structures for clean, top adsorption of $\mathrm{Na}$, and $\mathrm{Na}$-intercalation in graphene are shown in [(a)-(c)], respectively. Atoms are denoted in the same way as in Fig. 2. We observe that $\mathrm{Na}$ deposition increases the charge carrier density in top most graphene layer. The buffer layer, on the other hand is inert to the $\mathrm{Na}$ adsorption on top graphene layer but affected by $\mathrm{Na}$ intercalation.

age. The additional interaction between $\mathrm{Na}$ ions and graphene-buffer dipole layer is responsible for the increase in $\mathrm{Na}$ adsorption energy. Figure 3(c) shows the atomic structure of $\mathrm{Na}$ intercalation into graphene-buffer layers and corresponding band structures. Contrary to the top layer adsorption in Fig. 3(b), we find that the free electron band of $\mathrm{Na}$ becomes completely empty and the buffer layer $\pi$ band is partially occupied. Calculated cohesive energy shows that the $\mathrm{Na}$ intercalation is energetically more stable than $\mathrm{Na}$ adsorption on the top graphene layer by about $0.73 \mathrm{eV}$ per unit cell. Still the initial intercalation of Na should overcome the kinetic barrier, and a substantial heating will be needed to initiate the intercalation. Once Na intercalation occurs, both the buffer and top-most graphene can conduct. As the wave functions of the Fermi-level states at top graphene do not extend to the buffer layer, the immediate effect of bufferlayer charging upon intercalation will be mostly electrostatic. The gate electric field, on the other hand, may exhibit a different effect when $\mathrm{Na}$ intercalation occurs.
In summary, we studied the electronic property of epitaxial graphene with $\mathrm{Na}$ intercalation or adsorption. A strong coverage dependence is found both in $\mathrm{Na}$ adsorption energy and the charge transfer from $\mathrm{Na}$ to graphene that decrease as an inverse of Na coverage at low coverage. The buffer layer is found to be inert to Na adsorption on top layer graphene but become conducting upon $\mathrm{Na}$ intercalation. Our calculations indicate that the conduction of epitaxial graphene with $\mathrm{Na}$ doping can exhibit a contrasting behavior depending on whether $\mathrm{Na}$ adsorbs on top layer graphene or intercalates into buffer and graphene layers.

This work was supported by the Korea Science and Engineering Foundation (KOSEF) funded by the Korea government (MEST) under Grant No. R01-2008-000-20020-0. Authors thank C. Hwang and J. Chung for helpful discussions.

${ }^{1}$ P. R. Wallace, Phys. Rev. 71, 622 (1947).

${ }^{2}$ J. C. Slonczewski and P. R. Weiss, Phys. Rev. 109, 272 (1958).

${ }^{3}$ K. S. Novoselov, A. K. Geim, S. V. Morozov, D. Jiang, M. I. Katsnelson, I. V. Grigorieva, S. V. Dubonos, and A. A. Firsov, Nature (London) 438, 197 (2005).

${ }^{4}$ Y. B. Zhang, Y. W. Tan, H. L. Stormer, and P. Kim, Nature (London) 438 , 201 (2005).

${ }^{5}$ Y. W. Son, M. L. Cohen, and S. G. Louie, Phys. Rev. Lett. 97, 216803 (2006).

${ }^{6}$ S. Y. Zhou, G. H. Gweon, A. V. Fedorov, P. N. First, W. A. De Heer, D. H. Lee, F. Guinea, A. H. C. Neto, and A. Lanzara, Nature Mater. 6, 770 (2007).

${ }^{7}$ K. S. Novoselov, A. K. Geim, S. V. Morozov, D. Jiang, Y. Zhang, S. V. Dubonos, I. V. Grigorieva, and A. A. Firsov, Science 306, 666 (2004).

${ }^{8}$ I. Forbeaux, J. M. Themlin, V. Langlais, L. M. Yu, H. Belkhir, and J. M. Debever, Surf. Rev. Lett. 5, 193 (1998).

${ }^{9}$ S. M. Choi and S. H. Jhi, Phys. Rev. Lett. 101, 266105 (2008).

${ }^{10}$ M. Caragiu and S. Finberg, J. Phys.: Condens. Matter 17, R995 (2005).

${ }^{11}$ M. S. Dresselhaus and G. Dresselhaus, Adv. Phys. 51, 1 (2002).

${ }^{12}$ M. L. Cohen, Phys. Scr. T1, 5 (1982).

${ }^{13}$ J. P. Perdew, K. Burke, and M. Ernzerhof, Phys. Rev. Lett. 77, 3865 (1996).

${ }^{14}$ G. Kresse and J. Hafner, Phys. Rev. B 49, 14251 (1994).

${ }^{15}$ W. Chen, H. Xu, L. Liu, X. Y. Gao, D. C. Qi, G. W. Peng, S. C. Tan, Y. P. Feng, K. P. Loh, and A. T. S. Wee, Surf. Sci. 596, 176 (2005).

${ }^{16}$ T. Ohta, F. El Gabaly, A. Bostwick, J. L. McChesney, K. V. Emtsev, A. K. Schmid, T. Seyller, K. Horn, and E. Rotenberg, New J. Phys. 10, 023034 (2008).

${ }^{17}$ S. Kim, J. Ihm, H. J. Choi, and Y. W. Son, Phys. Rev. Lett. 100, 176802 (2008).

${ }^{18}$ A. Mattausch and O. Pankratov, Phys. Rev. Lett. 99, 076802 (2007).

${ }^{19}$ F. Varchon, R. Feng, J. Hass, X. Li, B. N. Nguyen, C. Naud, P. Mallet, J. Y. Veuillen, C. Berger, E. H. Conrad, and L. Magaud, Phys. Rev. Lett. 99, 126805 (2007). 